\title{
Polymer Conformations and Diffusion Through a Monolayer of
}

\section{Confining Nanoparticles}

\section{SUPPORTING INFORMATION}

Eric J. Bailey ${ }^{1}$, Robert A. Riggleman ${ }^{2}$, and Karen I. Winey*1,2

${ }^{1}$ Department of Materials Science and Engineering and ${ }^{2}$ Department of Chemical and

Biomolecular Engineering, University of Pennsylvania, Philadelphia, Pennsylvania 19104, United States

*Author to whom correspondence should be addressed. Electronic address:

winey@seas.upenn.edu 


\section{$\underline{\text { I. Table of Simulation Parameters }}$}

Table S1: Table of simulations parameters including chain length $(\mathrm{N})$, NP size $\left(2 \mathrm{R}_{\mathrm{NP}}\right)$, box dimensions $\left(\mathrm{L}_{\mathrm{x}}, \mathrm{L}_{\mathrm{y}}, \mathrm{L}_{\mathrm{z}}\right)$, macroscopic diffusion coefficient in $\mathrm{z}$ direction $\left(\mathrm{D}_{\mathrm{z}}\right)$, and the maximum LJ time.

\begin{tabular}{|l|l|l|l|l|l|l|l|l|}
\hline Confinement & $\mathbf{N}$ & $\mathbf{2 R}_{\mathbf{N P}}$ & $\mathbf{R}_{\mathbf{g}} / \mathbf{R}_{\mathbf{N P}}$ & $\mathbf{L x}$ & $\mathbf{L y}$ & $\mathbf{L z}$ & $\mathbf{D}_{\mathbf{z}}$ & $\mathbf{L J}$ time \\
\hline Bulk & 50 & N/A & N/A & 22.744 & 22.744 & 22.744 & $8.7 \mathrm{E}-4$ & $8.3 \mathrm{E} 6$ \\
\hline 0.5 & 50 & 7 & 1 & 21.2 & 18.36 & 57.566 & $7.6 \mathrm{E}-4$ & $1.7 \mathrm{E} 7$ \\
\hline 0.75 & 50 & 7 & 1 & 24.8 & 21.48 & 42.167 & $7.7 \mathrm{E}-4$ & $7.8 \mathrm{E} 6$ \\
\hline 1 & 50 & 7 & 1 & 28.4 & 24.6 & 57.4 & $8.2 \mathrm{E}-4$ & $1.2 \mathrm{E} 7$ \\
\hline 2 & 50 & 7 & 1 & 21.4 & 37.07 & 47.4 & $8.5 \mathrm{E}-4$ & 9E6 \\
\hline 1 & 50 & 14 & 0.5 & 21.4 & 18.53 & 53.9 & -- & $4.8 \mathrm{E} 6$ \\
\hline 1 & 50 & 3.5 & 2 & 21.2 & 36.72 & 56.1 & -- & $5.4 \mathrm{E} 6$ \\
\hline
\end{tabular}




\section{Polymer Density in Simulation Box}

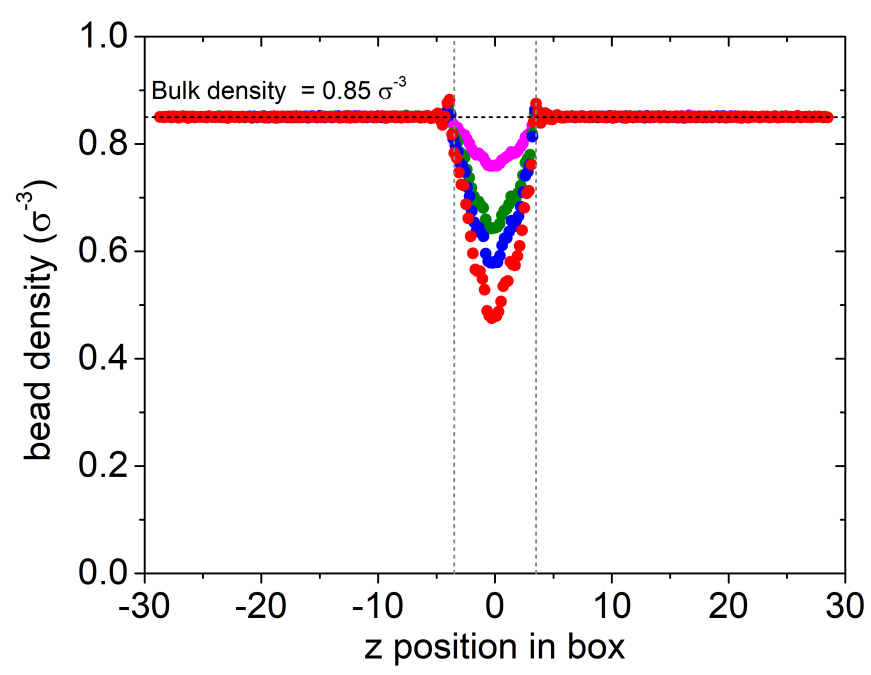

Figure S1: Local polymer density as a function of $\mathrm{z}$ position in the simulation box. Data presented are for $\mathrm{N}=50$ and $2 \mathrm{R}_{\mathrm{NP}}=7 \sigma$. Colors represent different levels of confinement: $I D / 2 \mathrm{R}_{\mathrm{g}}=0.5$ (red), 0.75 (blue), 1 (green), and 2 (magenta). 


\section{Random-walk Conformation Maps}

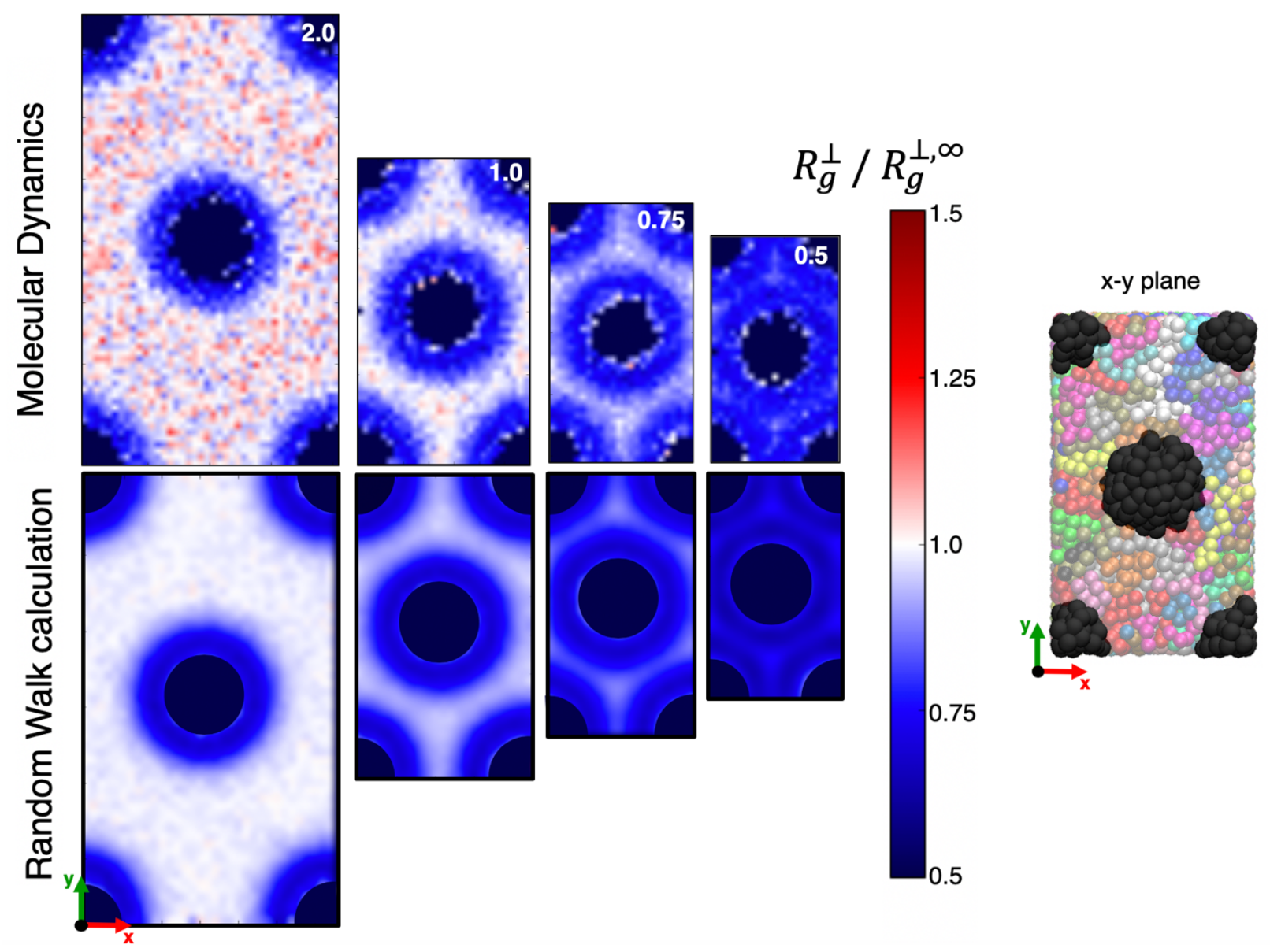

Figure S2: Conformation maps in $x-y$ plane through NP monolayer for various degrees of confinement (ID/2 $2 \mathrm{R}_{\mathrm{g}}$ ) obtained from MD simulations (top) and random walk calculations (bottom). NP representations in maps exclude conformations where the COM of the conformation is within the NP excluded volume. Data plotted are for $2 \mathrm{R}_{\mathrm{NP}}=7 \sigma\left(\sim 2 \mathrm{R}_{\mathrm{g}}\right)$ and $\mathrm{N}=50$ for MD and $\mathrm{n}$ $=83$ for random walk calculations. 


\section{Random-walk Conformations Around NPs}
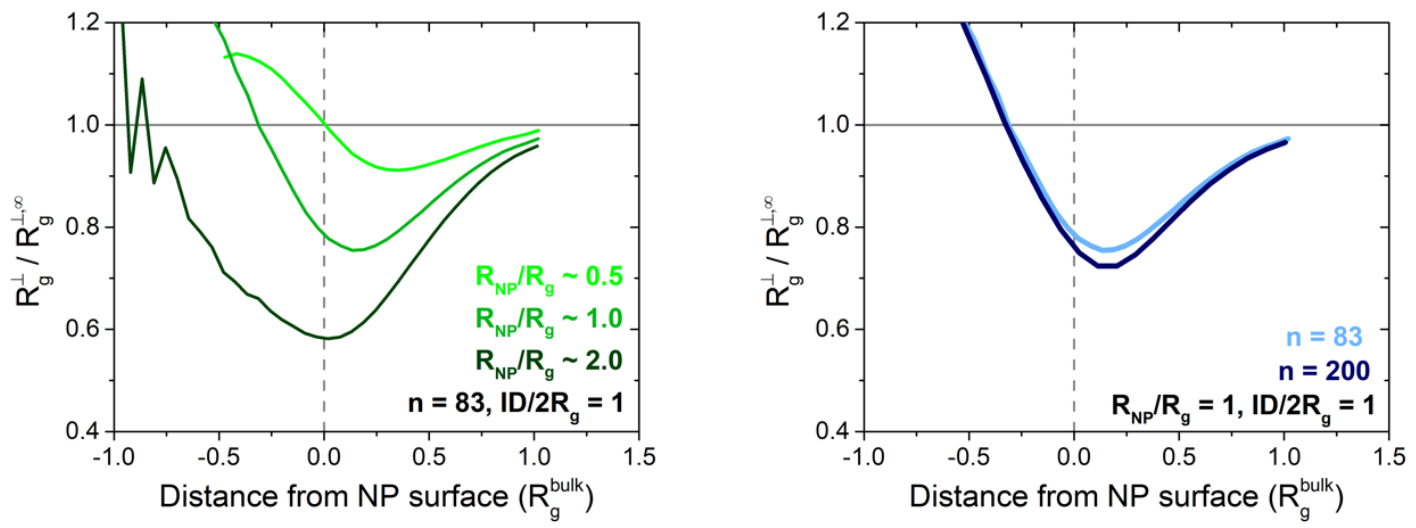

Figure S3: Conformation profile plotted as normalized perpendicular component of $\mathrm{R}_{\mathrm{g}}$ as a function of COM distance from the NP surface (in units of $\mathrm{R}_{\mathrm{g}}$ ) for different NP sizes (left) and chain lengths (right). 


\section{$\underline{\text { V. MSD of Bulk and PNC systems }}$}
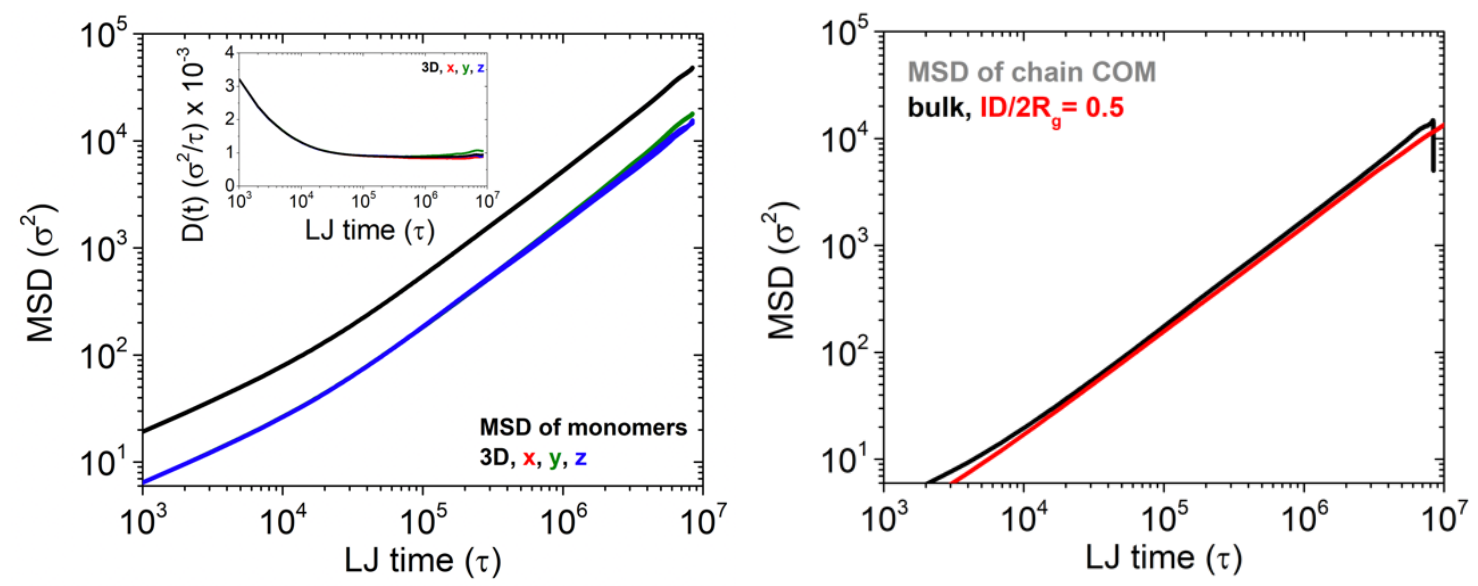

Figure S4: Macroscopic mean-squared displacement as a function of $\mathrm{LJ}$ time for bulk $\mathrm{N}=50$ chains (left). Comparison of MSD in z-direction for $\mathrm{N}=50$ chains in bulk and $\mathrm{ID} / 2 \mathrm{R}_{\mathrm{g}}=0.5$ with $2 \mathrm{R}_{\mathrm{NP}}=7 \sigma$ (right). 


\section{Diffusion Coefficient extracted from van Hove Distribution as a function of time}
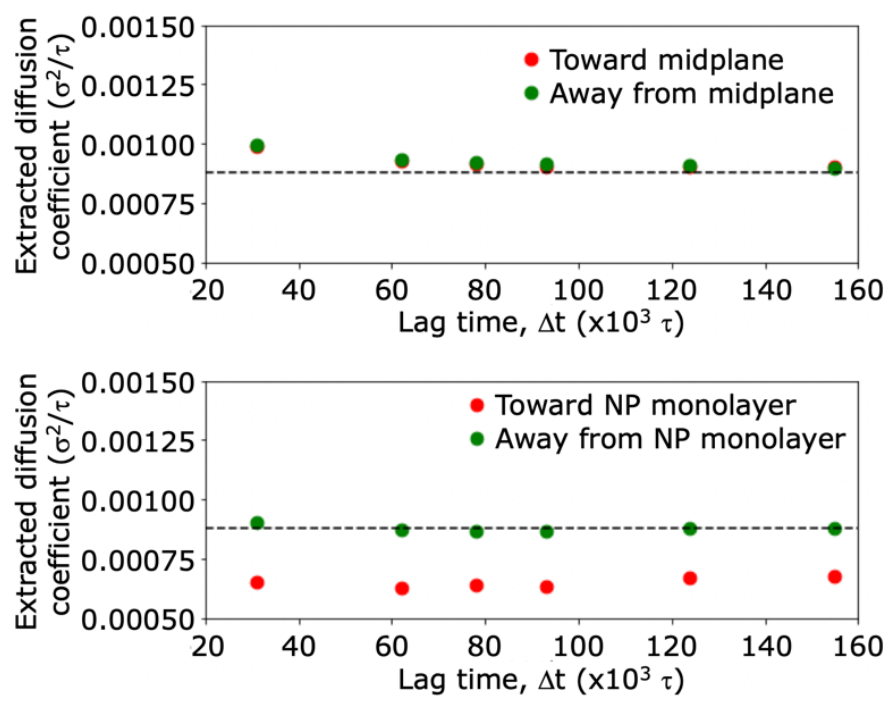

Figure S5: Extracted diffusion coefficient from van Hove distribution analysis as a function of time for bulk polymer (top) and ID/2 $\mathrm{R}_{\mathrm{g}}=0.5$ (bottom) for $\mathrm{N}=50$ chains, $2 \mathrm{R}_{\mathrm{NP}}=7 \sigma$, and $\mathrm{z}_{0}=4 \sigma$. Dashed lines represent macroscopic diffusion coefficient of bulk polymer. In bulk, the diffusion coefficient toward and away from the midline (i.e. where the NPs reside when they are present) overlap as expected and both equal the macroscopic diffusion coefficient. When the NP monolayer is present, the diffusion coefficient toward the NP monolayer is lower than both away from the NP monolayer and the bulk value, as discussed in the text. 


\section{VII. van Hove Distributions for representative $\mathrm{z}_{0}$}
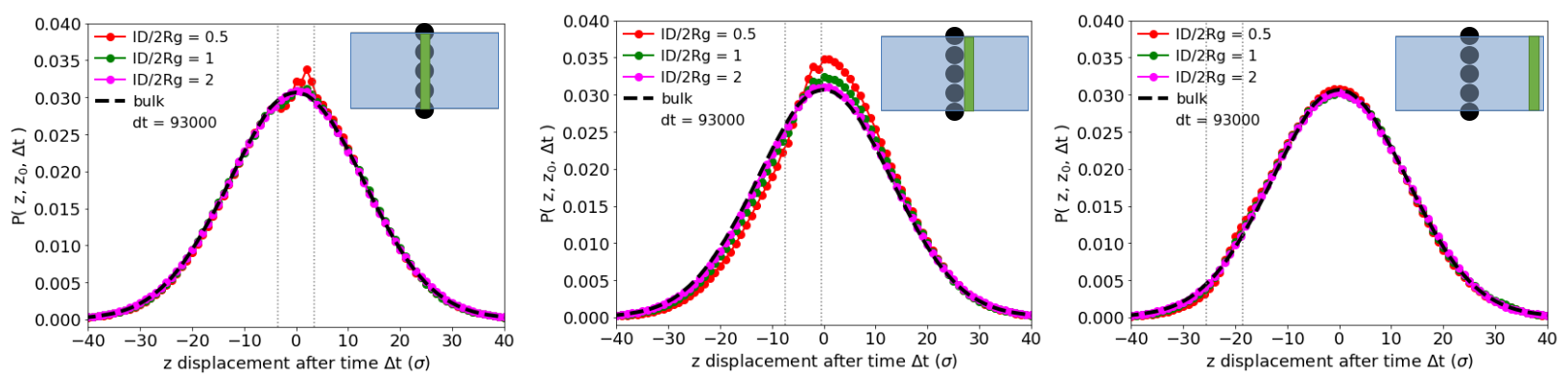

Figure S6: Van hove distributions for bulk and confined systems for several starting locations, as depicted in the insets, and $\Delta \mathrm{t}=93,000 \tau$. Figures include $\mathrm{z}_{0}=0$ (left), $\mathrm{z}_{0}=4$ (middle), and $\mathrm{z}_{0}=22$ (right), are obtained from systems with $\mathrm{N}=50$ and $2 \mathrm{R}_{\mathrm{NP}}=7 \sigma$, and are corrected for the NP volume as discussed in the main text. 


\section{Fitting of van Hove Distributions}

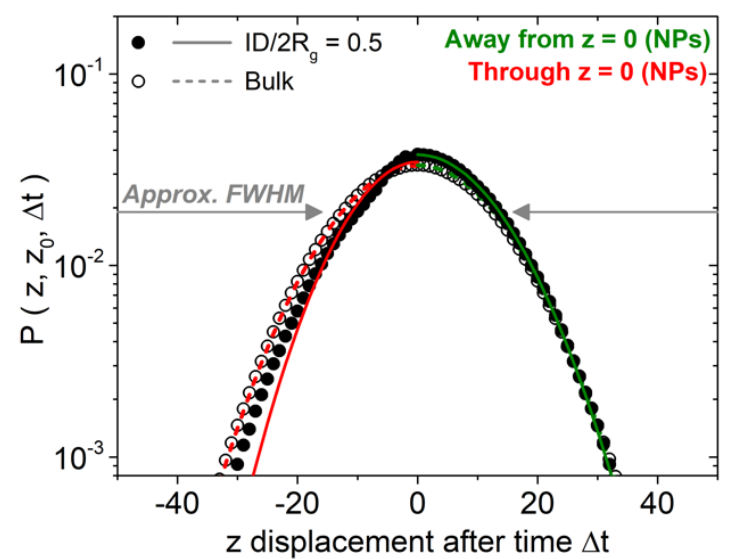

Figure S7: Corrected van Hove distribution of ID/2 $\mathrm{R}_{\mathrm{g}}=0.5$ (solid symbols) compared to bulk (open symbols) replicated from Figure $7 \mathrm{~b}$ of the manuscript but plotted with a $\log \mathrm{y}$-axis. Directionally fitted Gaussian profiles are shown in red and green lines. MD simulations used $\mathrm{N}=50$ and $2 \mathrm{R}_{\mathrm{NP}}=7 \sigma$. Both fits to bulk and the fit away from the NP monolayer in the PNC accurately represent the data. Although the fit to PNC data through the NP monolayer underrepresents the data at long distances, it accurately captures the FWHM and area under the curve of the data set, as discussed in the manuscript. 